\title{
Efeitos da alteração do limite de exposição ocupacional à sílica cristalina no processo de seleção de respiradores
}

\author{
An update in the threshold limit value for crystalline silica \\ and its effects on respirator selection
}

André Lomonaco Beltrame

Mestrando, Departamento de Engenharia de Minas e Petróleo,

Escola Politécnica,

Universidade de São Paulo

E-mail:deko@usp.br

\section{Sérgio Médici de Eston \\ Prof. Titular, Departamento de Engenharia de Minas e de Petróleo, Escola Politécnica, \\ Universidade de São Paulo E-mail:smeston@usp.br}

\section{Wilson S. Iramina}

Prof. Dr., Departamento de Engenharia de Minas e de Petróleo, Escola Politécnica,

Universidade de São Paulo E-mail:wilson.iramina@poli.usp.br

\section{Ana Carolina Chieregati \\ Professora, Dra., Departamento de Engenharia de Minas e de Petróleo, \\ Escola Politécnica, Universidade de São Paulo \\ E-mail: ana.chieregati@poli.usp.br}

Ivan Koh Tachibana

Departamento de Engenharia de Minas e de Petróleo, Escola Politécnica, Universidade de São Paulo E-mail: ivan.tachibana@poli.usp.br

\section{Resumo}

O objetivo desse trabalho foi investigar o processo de seleção de equipamentos de proteção respiratória e verificar a existência de mudanças nesse processo quando o limite de exposição ocupacional de uma substância é alterado. Utilizaram-se, como base de dados, dois estudos anteriormente realizados em empresas mineradoras da região metropolitana da cidade de São Paulo, selecionando-se, como contaminante, a sílica cristalina devido à sua abrangência e à gravidade das doenças relacionadas à sua exposição. Os fatores de proteção atribuídos aos equipamentos fornecidos pelas empresas estudadas foram comparados com os requeridos pelas atividades e com a metodologia do programa de proteção respiratória da FUNDACENTRO. Os resultados mostraram que, até 2005, algumas empresas estavam fornecendo equipamentos inadequados e que, a partir de 2006, ano em que foi alterado o limite de exposição, todas as empresas estudadas forneciam proteção insuficiente. Concluiu-se ser oportuna a criação de um portal na internet onde a seleção dos EPIs possa ser feita, de maneira atualizada, a partir das informações fornecidas pelas empresas.

Palavras-chave: Mineração, sílica cristalina, EPI, respiradores, TLV.

\begin{abstract}
The purpose of this study was to investigate the respiratory protective device selection process and to identify changes in this process when an exposure limit value is updated. Two previous studies conducted in mining industries in the metropolitan area of São Paulo were put through the respiratory protective device selection process. The protection factors of the equipment provided by the companies were compared with the required protection factors and with the FUNDACENTRO's respiratory protection program. The results showed that until 2005, some companies were providing inadequate protection, and after the change in crystalline silica exposure limit value in 2006, all the analyzed companies were providing inadequate respirators. This study suggests that there is an opportunity to create a web portal, where the selection process can be done by the companies with updated information.
\end{abstract}

Keywords: Mining, crystalline silica, PPE, respirators, TLV. 


\section{Introdução}

O Equipamento de Proteção Individual (EPI) pode ser considerado como a última barreira entre uma condição perigosa e o trabalhador. Segundo a Norma Regulamentadora número 6 (NR-6) (Brasil, 2009), a utilização dos EPIs deve ser restrita às situações nas quais as medidas de proteção coletiva não ofereçam proteção completa, e, também, quando tais medidas estiverem em fase de estudo ou em situações de emergência. A escolha e o rigor nas especificações do EPI são essenciais, devendo atender à legislação Brasileira e proteger os usuários, conservando a saúde dos mesmos.

As considerações sobre os EPIs não se limitam a proteger, ou não, o trabalhador. O uso impróprio do EPI pode comprometer o desempenho, a segurança, o conforto físico- emocional e a comunicação dos trabalhadores (Roberge, 2008). Os profissionais responsáveis pela escolha dos EPIs, como os integrantes do Serviço Especializado em Engenharia de Segurança e Medicina do Trabalho (SESMT) e da Comissão Interna de Prevenção de Acidentes (CIPA), acumulam muitas outras tarefas (identificar agentes nos processos de trabalho, participar da implementação e do controle da qualidade das medidas de prevenção necessárias, etc.). A escolha dos equipamentos de proteção deve ser revista, frequentemente, pois ocorrem:

- Mudanças no processo produtivo ou nos equipamentos: possível alteração do nível de exposição, introdução de novos contaminantes ou retirada de contaminantes (substituição de um reagente).

- Mudanças na legislação: alteração do nível de exposição máximo permitido para a jornada de trabalho. Verificou-se que, no período de 1996 a 2008, foram propostas mais de 160 alterações nos Threshold Limit Values (TLVs) da American Conference of Governmental Industrial Hygienists (ACGIH).

- Desenvolvimento de novas tecnologias: equipamentos que possam promover maior conforto com a mesma proteção (como máscaras e filtros mais leves).

- Avanço no processo seletivo dos equipamentos: o conhecimento científico para a seleção dos equipamentos pode alterar a lista de equipamentos adequados (exemplo: método I e método II do National Institute for Occupational Safety and Health (NIOSH) para o cálculo do Noise Reducion Rating (NRR) de protetores auditivos).

- Mudanças nos equipamentos de proteção: fechamento de empresa fabricante, descontinuidade de linha de equipamentos e lançamento de novos produtos (desde o ano de 1997 foram emitidos, aproximadamente, $21 \mathrm{mil}$ certificados de aprovação, para todos os tipos de EPI, pelo Ministério do Trabalho e Emprego, MTE).

Algumas dessas mudanças podem ser consideradas diretamente pelo profissional responsável pela seleção de EPIs, como as mudanças no processo produtivo, ou a troca de um equipamento. Entretanto outros fatores só podem ser considerados pelos higienistas e outros profissionais da área de segurança e saúde que se mantenham atualizados (adquirindo a publicação anual da ACGIH, verificando alterações na legislação, acompanhando o mercado dos equipamentos de proteção, etc.). Surge, então, a necessidade de se criar uma forma centralizada de atualização do conhecimento relacionado à escolha dos EPIs, para que os responsáveis por sua escolha possam estar sempre informados e atualizados das mudanças ocorridas, facilitando essa tarefa e, assim, mantendo os trabalhadores protegidos e suas empresas em conformidade com os requerimentos legais.

\section{Objetivo}

O objetivo desse trabalho é analisar o processo de seleção de equipamentos de proteção respiratória, quando há alteração no limite de exposição de um contaminante, utilizando-se, como base de dados, dois estudos de caso publicados.

\section{Revisão bibliográfica}

A NR-6 considera como Equipamento de Proteção Individual todo dispositivo ou produto de uso individual utilizado pelo trabalhador, destinado à proteção de condições perigosas suscetíveis de ameaçar a segurança e a saúde no trabalho (Brasil, 2009).

Os efeitos da sílica cristalina no corpo humano incluem a silicose, que é considerada a principal doença ocupacional pulmonar no Brasil (Goelzer \& Handar, 2002). É uma doença pulmonar crônica e incurável, com uma evolução progressiva e irreversível, que pode causar falta de ar, fadiga, dor peitoral e falência respiratória. Estudos mostram que a sílica cristalina está associada ao agravamento de outras doenças respiratórias e pode estar ligada ao câncer de pulmão (Rice et al., 2008).

Com a publicação da Instrução Normativa - I.N nº1 de 11/4/1994, pelo MTE, tornaram-se obrigatórias as recomendações do Programa de Proteção Respiratória (PPR) da FUNDACENTRO para todas as empresas onde estejam presentes condições perigosas para o trato respiratório que não foram eliminadas com a implantação de medidas de proteção coletiva ou durante a implantação das mesmas.

De acordo com o item 4.2.2.2 do Programa de Proteção Respiratória da FUNDACENTRO (FUNDACENTRO, 2002), é necessário calcular o fator de proteção requerido (FPR) através da equação (1):

$F P R_{j}=\frac{C_{j}}{L E}$

Onde:

$F P R_{j}$ : Fator de Proteção Requerido do contaminante $j$

$C_{j}$ : Concentração do contaminante $j$

LE: Limite de Exposição Ocupacional (aqui o TLV da ACGIH)

Define-se fator de proteção atribuído (FPA) como o nível mínimo de proteção respiratória que se espera alcançar no local de trabalho, para uma percentagem especificada de usuários 
treinados. Segundo o FPA, o trabalhador qye esteja sob risco deve usar um respirador apropriado (ou classe de respirador), em bom estado e ajustado corretamente ao rosto (ABNT, 1999). Segundo Torloni e Vieira (2003), afirmar que o fator de proteção atribuído (FPA) é 10 significa que para 95\% dos usuários a concentração inalada do contaminante será no mínimo 10 vezes menor que a do ambiente. Para atingir o FPA, seriam necessárias as seguintes condições:

- O respirador deve possuir tamanho e formato adequado para cada usuário.

- O respirador deve ser selecionado levando-se em consideração o ensaio de vedação.

- O usuário deve não omitir o uso.

- O usuário deve estar bem treinado.

- O respirador deve estar em perfeitas condições de uso.

Pode-se calcular o efeito da omissão de uso de um trabalhador durante a jornada, utilizando-se a fórmula do Fator de Proteção Efetivo (FPE) (Torloni \& Vieira, 2003):

$$
F P E=\frac{T}{\frac{T u}{F P T}+T o}
$$

Onde:

$T=$ Tempo de exposição

$T u=$ Tempo que o respirador foi utilizado

$T o=$ Tempo de omissão

FPT = Fator de Proteção no local de Trabalho

\section{Materiais e métodos}

Para o desenvolvimento desse trabalho, foram consultados os Limites de Exposição Ocupacional (TLVs ${ }^{\circledR}$ ) para Substâncias Químicas e Agentes Físicos \& Índices Biológicos de Exposição (BEIs ${ }^{\circledR}$ ) da ACGIH dos anos de 1996 a 2008. Foram utilizados, como objeto da seleção dos respiradores, estudos de caso de uma empresa de mineração a céu aberto produtora de brita (Gruenzner, 2003) e de 22 marmorarias (Bon, 2006), todas elas da região metropolitana de São Paulo. Escolheu-se analisar apenas o caso da sílica cristalina pela abrangência da indústria brasileira que manipula este contaminante e pela severidade dos efeitos causados no organismo dos trabalhadores expostos. As últimas alterações da ACGIH para os TLVs para a sílica cristalina foram introduzidas no ano de 2006 e, por essa razão, foi realizado o cálculo do fator de proteção requerido para cada atividade com base nos valores de TLV antes e após essa alteração. Uma vez calculados os fatores de proteção requeridos para as funções, compararam-se esses valores com os fatores de proteção atribuídos aos equipamentos disponibilizados pela empresa, determinando se os mesmos eram adequados ou não. Utilizando-se a metodologia do programa de proteção respiratória da FUNDACENTRO, foram determinados os equipamentos adequados para as funções, antes (2005) e após a alteração do limite (2006), comparando-se esses equipamentos com os fornecidos pelas empresas estudadas.

\section{Resultados}

No estudo de caso feito por Gruenzner, as 4 maiores concentrações foram de $0,67,0,33,0,32$ e $0,28 \mathrm{mg} / \mathrm{m}^{3}$ e, no levantamento feito por Bon (Bon, 2006), foram 0,47 e $0,36 \mathrm{mg} / \mathrm{m}^{3}$. Foram selecionados os maiores valores, pois, nas empresas analisadas eles se relacionavam às atividades que exigiam a utilização dos equipamentos de proteção respiratória. No ano de 2005, o TLV para sílica cristalina era de $0,050 \mathrm{mg} / \mathrm{m}^{3}$ (ACGIH, 2005), em 2006, houve redução desse limite de exposição ocupacional para $0.025 \mathrm{mg} / \mathrm{m}^{3}$, valor que é utilizado desde então.

O valor do FPR depende da atividade que está sendo analisada (através da concentração do contaminante no local) e do limite de exposição ocupacional utilizado. A Tabela 1 a seguir foi construída calculando-se o FPR para cada função, utilizando-se os TLVs de 2005 e de 2006.
Considerando-se o limite de exposição ocupacional de 2005, a maioria das atividades apresenta FPR abaixo de 10, valor este que, segundo a Tabela 3 do PPR (FUNDACENTRO, 2002), exigiria a utilização, no mínimo, de respirador purificador de ar não motorizado, tipo peça semifacial filtrante (PFF). Já para a atividade de "Marteleiro 1", é indicado respirador purificador com peça facial inteira ou um respirador purificador motorizado com peça semifacial, ambos com filtro P2 ou P3. Nas empresas analisadas, os equipamentos selecionados eram respiradores purificadores de ar tipo peça semifacial filtrante (PFF), indicação que está de acordo com o necessário para 5 das 6 funções analisadas e em desacordo para a atividade "Marteleiro 1".

Atualizando-se os índices do limite de exposição ocupacional, pode-se verificar que, em todas as atividades, o FPR está acima de 10, portanto devemse utilizar os respiradores purificadores de ar com peça facial inteira e filtro P2 ou P3, respiradores purificadores de ar motorizados com peça semifacial e filtro P2, respiradores de linha de ar comprimido com fluxo contínuo e peça semifacial ou respiradores de linha de ar comprimido de demanda com pressão positiva e peça semifacial. Em algumas situações, seria inviável a utilização desses equipamentos, devido ao calor, à impossibilidade física de fornecer linha de ar comprimido e à necessidade de boa visibilidade para execução das tarefas. Seria, então, necessário que a empresa verificasse qual seria a melhor maneira de se reduzir a exposição, seja pela mudança do processo (utilização de spray de água, troca dos equipamentos), seja pela diminuição do tempo de exposição.

\section{Discussão}

Observa-se que a redução do limite de exposição para metade do valor anterior tornou insuficiente a proteção dos EPIs de todas as atividades e um novo dimensionamento deveria ser feito conforme os requisitos do PPR da FUNDACENTRO. 
Tabela 1 - Cálculo dos FPRs.

\begin{tabular}{|c|c|c|c|c|c|}
\hline Atividade & $\begin{array}{c}\text { Concentração de } \\
\text { Sílica Cristalina } \\
\left(\mathrm{mg} / \mathrm{m}^{3}\right)\end{array}$ & $\begin{array}{l}\operatorname{TLV}(2005) \\
\left(\mathrm{mg} / \mathrm{m}^{3}\right)\end{array}$ & $\begin{array}{c}\operatorname{TLV}(2006) \\
\left(\mathrm{mg} / \mathrm{m}^{3}\right)\end{array}$ & FPR (2005) & FPR (2006) \\
\hline \multicolumn{6}{|c|}{ Estudo de Caso de Gruezner - Empresa produtora de brita } \\
\hline Acabador de Ardósia & 0,47 & \multirow{2}{*}{0,05} & \multirow{2}{*}{0,025} & 9,4 & 18,8 \\
\hline Acabador de Granito & 0,36 & & & 7,1 & 14,2 \\
\hline \multicolumn{6}{|c|}{ Estudo de Caso de Bon - Marmoraria } \\
\hline Marteleiro 1 & 0,67 & \multirow{4}{*}{0,05} & \multirow{4}{*}{0,025} & 13,4 & 26,8 \\
\hline Operador de Perfuratriz & 0,33 & & & 6,6 & 13,2 \\
\hline Marteleiro 2 & 0,32 & & & 6,4 & 12,8 \\
\hline Operador do Britador & 0,28 & & & 5,6 & 11,2 \\
\hline
\end{tabular}

As alterações podem ser maiores do que a redução pela metade observada para sílica. No caso do berílio, por exemplo, houve alteração do limite de $0,002 \mathrm{mg} / \mathrm{m}^{3}$ para $0,00005 \mathrm{mg} / \mathrm{m}^{3}$, representando uma redução de 40 vezes (ACGIH, 2008).

Nos levantamentos de Gruezner e Bon, mesmo antes da alteração do limite de exposição, pode-se questionar se os trabalhadores estavam protegidos. No caso do acabador de ardósia, o FPR foi calculado em 9,46 e o EPI utilizado era respirador purificador de ar com máscara tipo semifacial, cujo FPA é 10.

Esses valores estão muito próximos e, caso o trabalhador não utilizasse o respirador por 10 minutos durante a jornada de trabalho de 6 horas, com FPA de 10, calcula-se:

$F P E=\frac{360}{\frac{250}{10}+10}=8$

O trabalhador estaria exposto a uma concentração acima do limite ocupacional, pois o FPE de 8 é inferior ao FPR calculado de 9.46.

Essa exposição pode ser agravada pelas condições dos equipamentos, pela falta de vedação ou acomodação na face do usuário. Ferreira et al. (2008) observaram que $75 \%$ dos trabalhadores registrados, em seus estudos, utilizavam EPIs inadequados, além de os mesmos ficarem depositados, muitas vezes, no chão. Essa observação indica a falta de percepção do risco pelos trabalhadores, consequência da falta de treinamento dado pelas empresas em relação à utilização e manutenção dos EPIs. Pode-se discutir, ainda, a validade da indicação de equipamentos de proteção que não sejam descartáveis, pois exigiria dos trabalhadores um maior cuidado, trazendo à tona a necessidade de se realizar um estudo para determinação da melhor forma de reduzir a exposição dos trabalhadores.

\section{Conclusões}

No Brasil, o número estimado de trabalhadores potencialmente expostos a poeiras contendo sílica é de 6 milhões (Goelzer \& Handar, 2002). Observouse, durante a pesquisa feita para esse trabalho, que as empresas não estão dimensionando corretamente os EPIs e, muitas vezes, se satisfazem com o fato de fornecer qualquer máscara ao trabalhador, mesmo que esta não o proteja completamente. Outras empresas não tinham conhecimento das atualizações dos valores, nem dos lançamentos de novos equipamentos de proteção. A falta de informações, a utilização incorreta e o dimensionamento incorreto dos equipamentos de proteção respiratória (caso da sílica) podem ser traduzidos diretamente em um custo socioeconômico. Este fica demonstrado através dos benefícios concedidos pela Previdência Social, que, no período de 1998 a 2003, chegou ao valor de $\mathrm{R} \$ 127.300 .000,00$ (Oliveira, 2007).

Conclui-se que é interessante a criação de uma forma centralizada de atualização dos dados e da metodologia de seleção de EPIs. Propõe-se que seja criado um portal na internet onde os engenheiros de segurança, técnicos de segurança e higienistas ocupacionais possam acessar informações de maneira simples e organizada, apoiando o processo de seleção de EPIs em conhecimento atualizado. As informações sobre equipamentos disponíveis, limites atuais e mudanças no conhecimento técnico, seriam revisadas pela equipe do portal, cabendo ao usuário apenas se cadastrar e responder às questões sobre os contaminantes encontrados e atividades realizadas na sua empresa, recebendo, ao final, uma lista com os equipamentos disponíveis no mercado que seriam adequados para as situações apresentadas. Esse trabalho utilizou, como exemplo, apenas o caso da sílica cristalina, existindo, ainda, uma infinidade de contaminantes presentes 
André Lomonaco Beltrame et al.

na indústria mineral que estão sujeitos ao mesmo raciocínio aqui apresentado, multiplicando o tamanho e a abrangência dos problemas apontados no processo de atualização da escolha dos EPIs.

\section{Referências}

\section{bibliográficas}

ACGIH. TLVs and BEIs. Cincinnati: 2005. $224 \mathrm{p}$.

ACGIH. TLVs and BEIs. Cincinnati: 2008. $274 \mathrm{p}$.

ASSOCIAÇÃO BRASILEIRA DE NORMAS TÉCNICAS. NBR-12543:

Equipamentos de proteção respiratória - terminologia. Rio de Janeiro: 1999. 45p.

BON, A.M.T. Exposição ocupacional à sílica e silicose entre trabalhadores de marmorarias, no municipio de São Paulo. São Paulo: Faculdade de Saúde Pública, Universidade de São Paulo, 2006. 323 p. (Tese de Doutorado).

BRASIL. Ministério do Trabalho e Emprego. Norma Regulamentadora 6: equipamento de proteção individual. Brasília: 2009. 8 p.
FERREIRA, L.R et al. A silicose e o perfil dos lapidários de pedras semipreciosas em Joaquim Felício, Minas Gerais, Brasil. Caderno de Saúde Pública, Rio de Janeiro, v.24, n.7, p. 1517-1526, jul. 2008.

FUNDACENTRO. Programa de proteção respiratória: seleção e uso de respiradores. São Paulo: FUNDACENTRO, 2002. 130 p.

GOELZER, B; HANDAR, Z. Programa de eliminação da silicose. 2002. Disponível em: <http://www.fundacentro.gov.br/silicaesilicose>. Acesso em: 15 mar. 2009.

GRUENZNER, G. Avaliação da poeira de sílica: um estudo de caso em uma pedreira na região metropolitana de São Paulo. São Paulo: Escola Politécnica, Universidade de São Paulo, 2003. 110 p. (Dissertação de Mestrado).

OLIVEIRA, K. Controle da exposição à poeira em marmorarias: embasamento técnico para proposta de atuação de governo visando ao controle da exposição a poeiras e outros riscos no setor de beneficiamento de rochas ornamentais em marmorarias. Apresentado ao Programa de Eliminação da Silicose. 2007. Disponível em <http://www.saudeetrabalho. com.br/download/marmoarias-katia.pdf>. Acesso em 12 mar. 2009.

RICE, F.L et al. Crystalline silica exposure and lung cancer mortality in diatomaceous earth industry workers: a quantitative risk assessment. Occupational and Environmental Medicine, London, v. 58 p. 38-45, Jan. 2008.

ROBERGE, R. Evaluation of the rationale for concurrent use of N95 filterind facepiece respirators with loose-fitting powered air-purifying respirators during aerosol-generating medical procedures. American Journal of Infection Control, St. Louis, v.36, n.2, p. 135-141, Mar. 2008.

TORLONI, M., VIEIRA, A. V. Manual de proteção respiratória. São Paulo: ABHO, 2003. $520 \mathrm{p}$.

Artigo recebido em 25/05/2009 e aprovado em 22/10/2009.

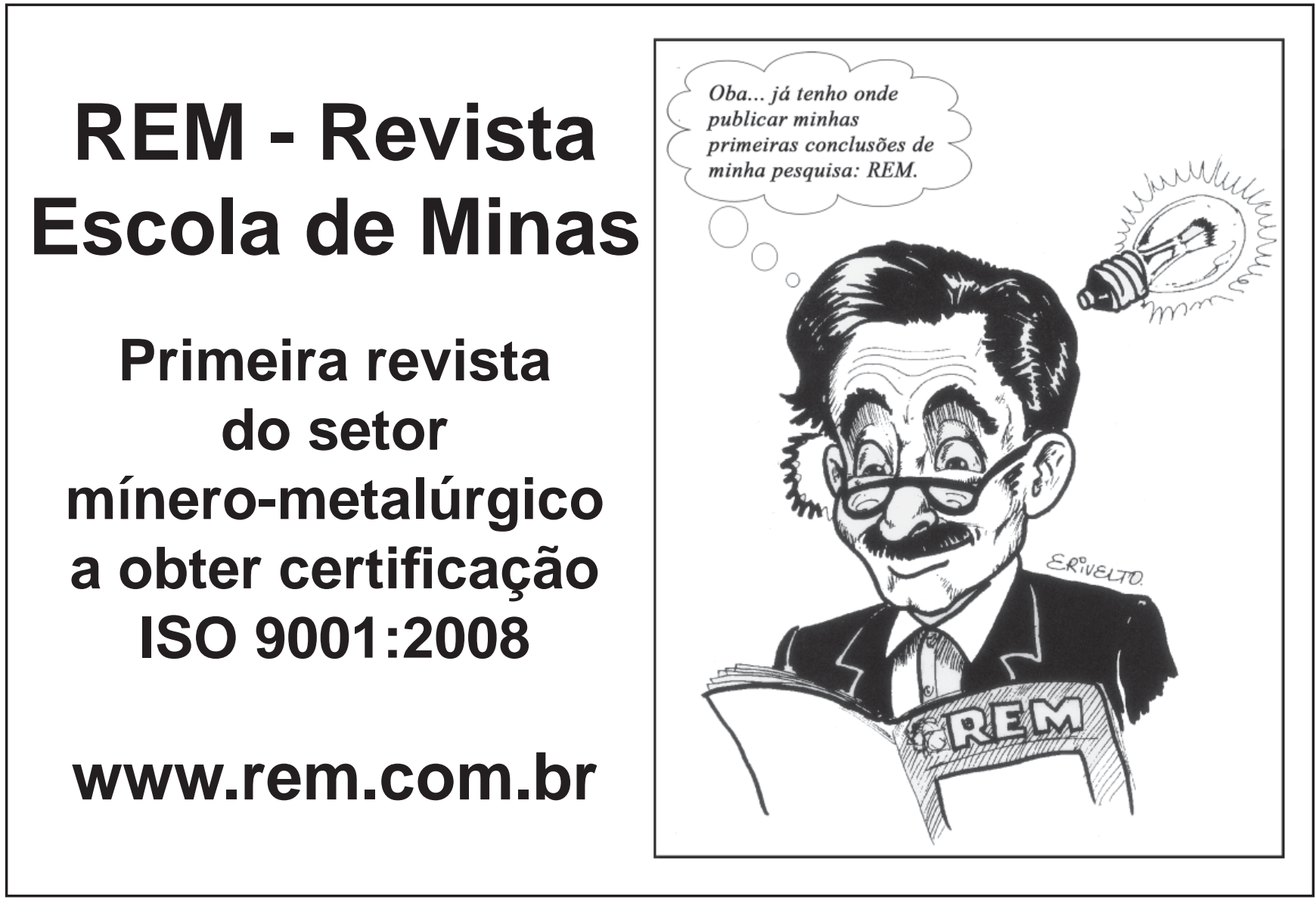

